\title{
NEAR-INFRARED GUIDED COLOR IMAGE DEHAZING
}

\author{
Chen Feng, Shaojie Zhuo, Xiaopeng Zhang, Liang Shen \\ Sabine Süsstrunk \\ Qualcomm Canada Inc. \\ EPFL
}

\begin{abstract}
Near-infrared (NIR) light has stronger penetration capability than visible light due to its long wavelength, thus being less scattered by particles in the air. This makes it desirable for image dehazing to unveil details of distant objects in landscape photographs. In this paper, we propose an improved image dehazing scheme using a pair of color and NIR images, which effectively estimates the airlight color and transfers details from the NIR. A two-stage dehazing method is proposed by exploiting the dissimilarity between RGB and NIR for airlight color estimation, followed by a dehazing procedure through an optimization framework. Experiments on captured haze images show that our method can achieve substantial improvements on the detail recovery and the color distribution over the existing image dehazing algorithms.
\end{abstract}

Keywords- Image dehazing, RGB, NIR, Detail transfer, Optimization, Airlight color

\section{INTRODUCTION}

Haze and mist significantly reduce the visibility in landscape photographs, which impact visual quality and bring difficulties for many computer vision applications [1]. Accurately estimating the airlight color and recovering the lost details in the color image is a fundamental and challenging problem in image processing.

Optically, the haze effect is due to the presence of particles in the atmosphere, with comparable size to the wavelength in the visible band (haze $\sim 0.1 \mathrm{um}$, mist $\sim 1 \mathrm{um}$ ), that absorb and scatter light. Reflected light from distant objects is attenuated and diffused by the particles. As the particle density increases, both color and details of distant scene will fade away. The advantage of deep penetration of the near-infrared (NIR) due to its long wavelength ( 1um) makes it possible to unveil the details, which could be completely lost in the visible band.

Image dehazing in general involves two tasks, removing the airlight color effect and recovering the lost details. State-of-the-art image dehazing algorithms [2][3][4], remove the haze based on a single RGB image. The core idea in these studies is to estimate the airlight color and the transmission map under certain assumption, such as dark channel prior, and then reconstruct the haze-free image based on the haze model. However, these algorithms suffer from an inherent problem of the single image input, which may not contain any scene details. Once the information is lost in the input image, it is very difficult for these algorithms to unveil the ground truth. In general, the recovered images at dense haze regions tend to be noisy and lack texture details.

Removing the airlight color is fundamental to image dehazing. Inaccurate estimation of the airlight color could result in unwanted color shift issues. Most current literatures [2][4] simply approximate the airlight color from the brightest region in the scene by assuming such regions are usually at infinity and have the most haze. However, this approach often fails by mistakenly selecting white objects (e.g., clouds) to estimate the airlight color. Essentially, it is hard to use single RGB image to differentiate the object with haze-like color (i.e., cloud) from the object under actual haze.

There are some other approaches removing the haze by exploiting the difference of two or more images of the same scene that have different properties. Nayar et al. use two images with the same scene captured with significant different mediums [1], which is impractical in reality and hard to deliver immediate results. Shwartz et al. use two images captured with different degrees of polarization by rotating a polarizing filter attached to the camera [5], which cannot handle dynamic scenes for most outdoor landscape pictures where objects like trees and clouds moving quickly.

The near-infrared spectrum can be easily acquired by using off-the-shelf digital cameras with minor modifications [6], or potentially through a single RGBN camera, in which multiple images with different properties can be captured simultaneously [7]. In [8], L. Schaul et al. first proposed to fuse the NIR detail information in the color image under the Weighted Least Squares (WLS) framework [9] without airlight color detection. The fundamental issue of this approach is that the texture details in the non-haze regions will also be boosted in luminance channel, thus resulting in color shifting artifacts.

Most previous works suffer from either detail lost or color shifting. Enlightened by the general haze model [3], however, we try to recover the color and transfer the details in one shot, aiming to provide a practical and complete solution. By exploiting dissimilarity of NIR and other color bands, we refined airlight color esitmation in a much meaningful way. By formulating image de-hazing as an optimization problem 
and introducing NIR gradient constraint, we succesfully removed haze effect by revealing accurate details and color, while leaving non-haze regions with minimum impact.

In this paper, we propose a novel image dehazing approach, using the images captured in both visible $(400-700 \mathrm{~nm})$ and near-infrared $(700-1100 \mathrm{~nm})$ bands. Our main contribution are in two folds:

- Propose an optimization framework to resolve image de-hazing problem guided with NIR gradient constraints.

- Refine airlight color estimation by exploiting the differences between NIR and RGB channels.

\section{PROBLEM FORMULATION}

Consider a general haze model [3],

$$
\mathbf{I}(x)=\boldsymbol{t}(x) \mathbf{J}(x)+(1-\boldsymbol{t}(x)) \mathbf{A},
$$

where for each pixel $x, \mathbf{I}(x)$ stands for the observed image; $\mathbf{J}(x)$ is the haze-free image; A represents for the global airlight color, which is a $3 \times 1$ vector in typical outdoor landscape photographs; $\boldsymbol{t}(x)$ is the medium transmission describing the portion of the light that is not scattered and reaches the camera. Therefore, by removing the haze, it is required to recover $\mathbf{J}$ given the color image $\mathbf{I}^{R G B}$ and the near-infrared image $\mathbf{I}^{N I R}$. For the brevity of discussion in this paper, we assume the pair of RGB and NIR images are well registered.

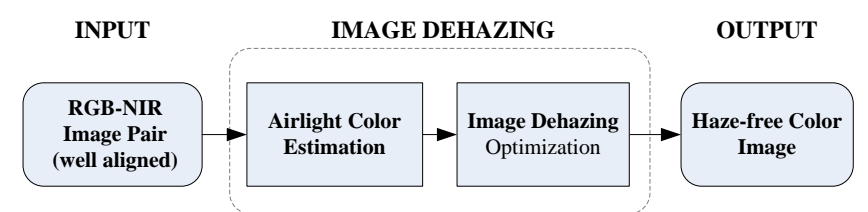

Fig. 1. The overview of the proposed image dehazing scheme.

We propose a two-stage dehazing scheme: an airlight color estimation stage by exploiting the dissimilarity between RGB and NIR; and an image dehazing stage by enforcing the NIR gradient constraint through an optimization framework. Fig. 1 shows the overall workflow of the proposed scheme.

\section{AIRLIGHT ESTIMATION}

In order to recover $\mathbf{J}$ as formulated in (1), the first step of our approach is to estimate the global airlight color $\mathbf{A}$. A commonly used idea in literatures is to approximate the airlight color from the most hazed region in the scene, where the transmission tends to be zero. However, this approach has limitation on scenarios with light haze. Inspired by [3], we consider that the transmission $t$ depends on the scene depth and the density of the haze, while the intrinsic color $\mathbf{J}$ depends on the illumination of the scene and the surface reflectance. Therefore, it is reasonable to assume that $\boldsymbol{t}$ and $\mathbf{J}$ are uncorrelated within a local patch. The idea we proposed here to estimate the airlight color consists of finding a local patch $\Omega$ with pixels having large similarities, followed by searching an airlight color that leads to the smallest correlation between $\boldsymbol{t}$ and $\mathbf{J}$.

One of our key innovations is to use NIR to help finding such a good local patch for the airlight color estimation. A good local patch should meet two major criteria: i) pixels within the patch should have intermediate level of haze so that both $\mathbf{J}$ and $\mathbf{A}$ contribute to the observed intensity value I in equation (1); ii) pixels within the patch should have similar properties (i.e., surface reflectance). Therefore, we first generate a haze map to find pixels that satisfy i), and then adopt RGB-NIR relationship to meet the criteria ii).

Consider particle scattering, we can make two observations. Firstly, haze increases the intensity value over all R, G and B channels and thus reduces the image contrast. In other words, the smallest intensity value over the three color channels infers the density of the haze. We refer to this observation as the haze prior. Secondly, since blue light has the shortest wavelength in the visible band compared with NIR, it is scattered more and thus changes quickly as depth increases. Therefore, the difference between the blue channel and the NIR channel can be used to refine the density of the haze. By considering the two observations, we define a haze map $\mathcal{H}$, as shown in (2), to indicate the density of the haze.

$$
\mathcal{H}=\min \left\{\min _{k \in\{R, G, B\}}\left(\mathbf{I}^{k}\right), \mathbf{D}\right\}, \mathbf{D}=\mathcal{N}\left\{\left|\mathbf{I}^{B}-\mathbf{I}^{N I R}\right|\right\},
$$

where $\mathcal{N}\{\cdot\}$ represents the max-min normalization. Therefore, large value in $\mathcal{H}$ infers small transmission.

Criterion i) requires the patch contains an intermediate level of haze. Therefore, we calculate the histogram of $\mathcal{H}$, and use the first valley $h$ as a reference to select a coarse patch region $\Omega_{c}$. Pixels within $\Omega_{c}$ should have similar haze density, and the similarity is defined by $\delta$,

$$
\Omega_{c}=\{x:|\mathcal{H}(x)-h| \leq \delta\}
$$

Within the coarse patch region, a refined local patch $\Omega$ is obtained by searching those connected pixels with similar color properties. Particulary, pixels that have similar NIR value and blue to NIR ratios are retained.

By using the pair of RGB and NIR images, we are able to infer the density of haze and thus, pixels that have large similarities are selected to generate a local patch, within which we search for an airlight color $\mathbf{A}$ that leads to the smallest correlation, measured by Pearson's correlation coefficient $\mathcal{C}$, between $\boldsymbol{t}$ and $\mathbf{J}$. Please refer to [3] for the detailed definition of $\mathcal{C}$. Specifically, we update A's components using the steepest decent method by minimizing the Equation (4). Fig. 2 illustrates the haze map $\mathbf{H}$, its 


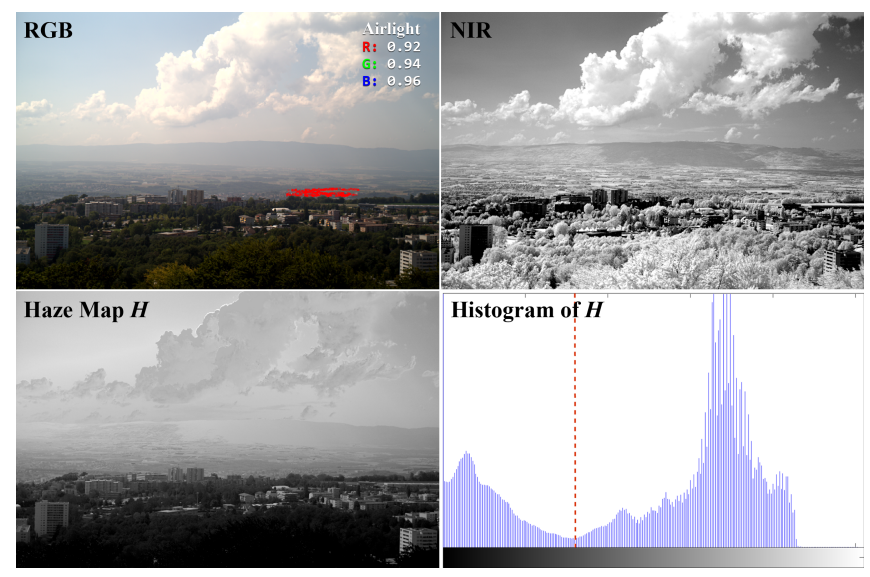

Fig. 2. Airlight color estimation. Top-left shows the original RGB image and the pixels selected to esitmate airlight color (makred with red cross). The pixels are selected based on first valley in histogram (bottom-right) of haze map H (bottom-left).

histogram and the selected patches for airlight color estimation.

$$
\mathbf{A}=\arg \min _{\forall(x) \in \Omega} \mathcal{C}(\mathbf{J}, \boldsymbol{t})^{2}
$$

\section{IMAGE DEHAZING}

The core to the algorithm is to formulate the whole image dehazing process as an optimization problem. This process includes estimating the haze-free color image and transferring details from the NIR to the color image.

\subsection{Initialization}

Divided by the estimated airlight color $\mathbf{A}$ at both sides in equation (1) together with the haze prior, it is easy to derive

$$
\boldsymbol{t}_{0}=1-\min _{k \in\{R, G, B\}}\left(\mathbf{I}^{k} / \mathbf{A}\right) .
$$

Since the haze prior may not lead $\min _{k \in\{R, G, B\}}\left(\mathbf{J}^{k} / \mathbf{A}\right)$ to be exactly equal to zero for each pixel, the transmission map $t_{0}$ calculated from (5) may tend to be smaller than the actual value. In addition, if we remove the haze thoroughly, the image may look unnatural. To compensate for this, we optionally keep a small amount of haze by introducing a weight $\omega$, which is large for distant objects and small for close objects, namely,

$$
\omega=\frac{1}{1+e^{-10(\mathcal{N}\{\mathcal{H}\}-0.5)}} *(a-b)+a,
$$

where $a$ and $b$ are pre-determined parameters $(a=0.6, b=$ 0.4 in our experiments).

In order to get a good initialization, we use the RGB input image as a guide to refine the transmission map $t_{0}$ for better edge alignment through guided filter [10]. Finally, the initialized $\mathbf{J}_{0}$ is calculated by,

$$
\mathbf{J}_{0}=\frac{\mathbf{I}-\mathbf{A}}{\max (\boldsymbol{t}, \varepsilon)}+\mathbf{A},
$$

where $\varepsilon$ equals to 0.1 in our experiments.

\subsection{Optimization Framework}

Recall our problem formulation in (1), removing the haze basically requires the recovery of $\mathbf{J}$ given $\mathbf{I}^{R G B}$ and $\mathbf{I}^{N I R}$. Statistically, this can be reformulated by finding the largest joint probability of $(\mathbf{J}, \boldsymbol{t})$ given $\mathbf{I}^{R G B}$ and $\mathbf{I}^{N I R}$. Based on Bayes' theorem, we further derive

$$
\begin{gathered}
P\left(\mathbf{J}, \boldsymbol{t} \mid \mathbf{I}^{R G B}, \mathbf{I}^{N I R}\right)=\frac{P\left(\mathbf{I}^{R G B}, \mathbf{I}^{N I R} \mid \mathbf{J}, \boldsymbol{t}\right) P(\mathbf{J}, \boldsymbol{t})}{P\left(\mathbf{I}^{R G B}, \mathbf{I}^{N I R}\right)} \\
\propto P\left(\mathbf{I}^{R G B} \mid \mathbf{J}, \boldsymbol{t}\right) P\left(\mathbf{I}^{N I R} \mid \mathbf{J}, \boldsymbol{t}\right) P(\mathbf{J}) P(\boldsymbol{t}) .
\end{gathered}
$$

Equation (8) contains four terms, in which $P\left(\mathbf{I}^{R G B} \mid \mathbf{J}, \boldsymbol{t}\right)$ corresponds to the haze model as defined in (1); $P\left(\mathbf{I}^{N I R} \mid \mathbf{J}, \boldsymbol{t}\right)$ represents the relationship between the color and the NIR images, which we refer to as the NIR constraint; $P(\mathbf{J})$ and $P(\boldsymbol{t})$ represent the color image prior and the transmission prior, respectively.

Based on the statistical analysis in (8), we find the optimal solution for $\mathbf{J}$ and $\boldsymbol{t}$ by solving the following optimization problem,

$$
\begin{aligned}
(\hat{\mathbf{J}}, \hat{\boldsymbol{t}}) & =\arg \min _{(\mathbf{J}, \boldsymbol{t})}\left\|\boldsymbol{t} \mathbf{J}+(1-\boldsymbol{t}) \mathbf{A}-\mathbf{I}^{R G B}\right\|^{2} \\
& +\lambda_{1} w\left|\nabla \mathbf{J}-\nabla \mathbf{I}^{N I R}\right|^{\alpha}+\lambda_{2}|\nabla \mathbf{J}|^{\beta}+\lambda_{3}\|\nabla \boldsymbol{t}\|^{2},
\end{aligned}
$$

where $\alpha, \beta \in(0,1)$. The first term in (9) comes from our haze model, which would produce the least noise in the haze-free image. The fact that NIR penetrates further than the visible band due to its long wavelength allows us to transfer the details from the NIR image to the color image. Therefore, we add a weighted gradient constraint in our second term. The last two terms are the smoothness priors for the natural image and the transmission map. In the weighted gradient constraint, we add large weights on distant objects and small weights on close objects. Thus, weight $\boldsymbol{w}$ is defined as

$$
\boldsymbol{w}=\frac{1}{1+e^{-10(0.5-\boldsymbol{t})}} .
$$

In equation (9), $\lambda_{2}$ and $\lambda_{3}$ are pre-determined parameters with small values ( 0.01 in our experiments), and $\lambda_{1}$ controls the level of detail transfer that comes from the NIR image.

By solving the optimization problem stated in (9) using Iteratively Reweighted Least Squares (IRLS) with initialized $\mathbf{J}_{0}$ and $\boldsymbol{t}_{0}$ derived from (7) and (5), we can recover the haze-free image $\mathbf{J}$ and the transmission map $\boldsymbol{t}$ simultaneously. 


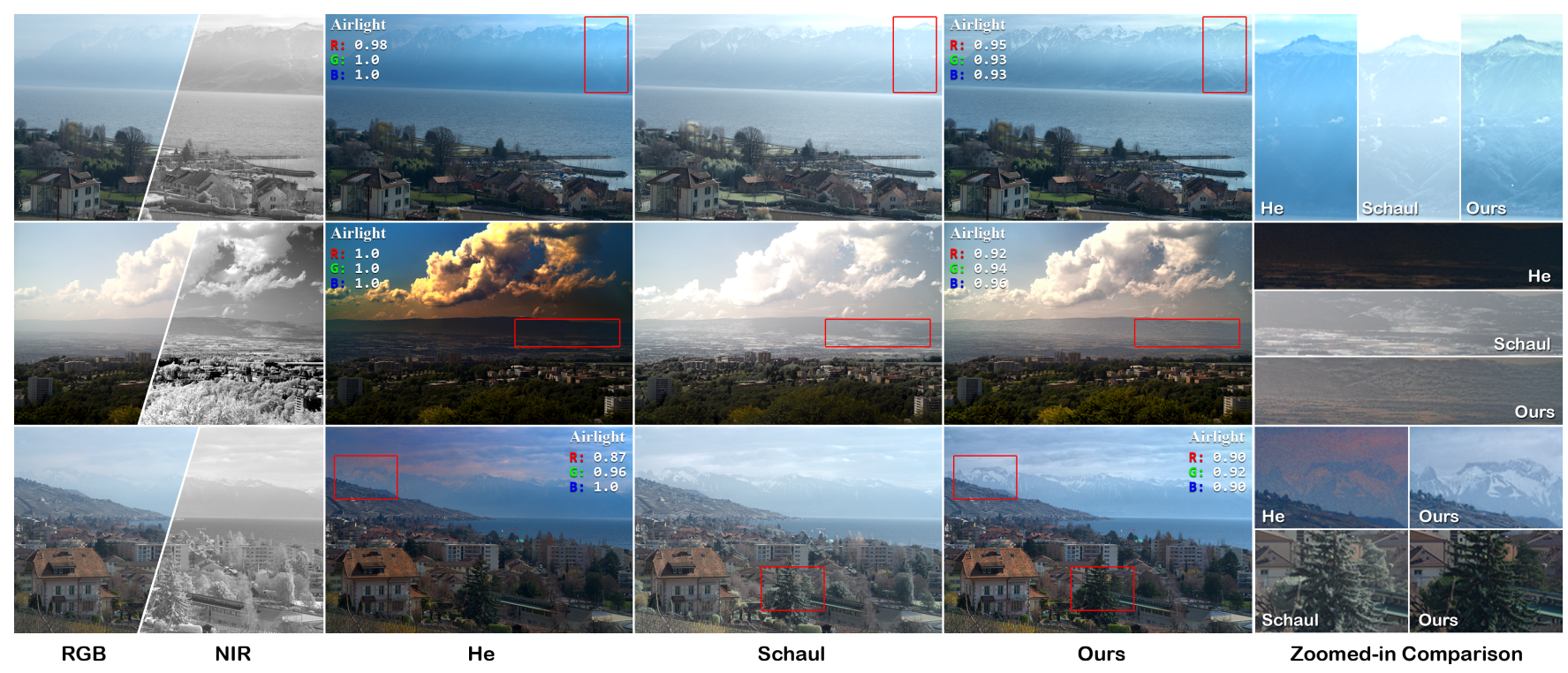

Fig. 3. The comparison with state-of-the-art image dehazing algorithms. Columns-wise from left to right: input RGB-NIR image pairs, single image dehazing results by [2], multiple image dehazing results by [8], our results and the zoomed-in comparison. The estimated airlight color of each method is indicated with values. Red rectangle areas are further zoomed-in and compared in the last column.

Different from traditional single image dehazing algorithms, our approach transfers detail information from the NIR image by enforcing the gradient constraint. By using the optimization framework, it handles well on the noise issue that comes from the division term in (7) when the transmission value $t$ is small. In addition, the transmission map $t$ can be different for each color channel, providing more accurate recovered results.

After the airlight veil is removed in $\hat{\mathbf{J}}$, the overall tone of the haze-free image could become darker than the input RGB image. In our final results, a local tone mapping is applied as a post-processing procedure to enhance the perceptual experience.

\section{EXPERIMENTAL RESULTS}

This section provides results of the proposed dehazing method applied on real captured image pairs using a modified commercial camera. Specifically, the visible band ranges from 400 to $700 \mathrm{~nm}$, and the NIR filter allows IR ranging from 700 to $1100 \mathrm{~nm}$ to pass. NIR and visible images of the same scene are obtained by placing alternatively a NIR or visible blocking filter on the lens. Potentially the pair of images can be captured simultaneously through a single RGBN camera in the future design [7].

Fig. 3 compares our dehazing result with the most recent state-of-the-art single/multiple image dehazing algorithms [2][8], where the first column shows the input image pairs. It is clear to see that NIR retains more details on distant objects than the corresponding color image. Compared with He's single image dehazing method [2], our result produces more details on distant objects. This is simply due to the fact that NIR can penetrate further than the visible band in the haze. Meanwhile, our result produces a haze free image with much less noise on distant objects. This benefit comes from the first term in our optimization framework (9). Furthermore, our result preserves the original illumination of the scene. Comparing to Schaul et al.'s work [8], since our approach adopts the haze model, we can not only selectively enhance the details based on the transmission map, but also well recover the original color of the scene.

From the experiments, it takes around 60 seconds to process a 1 megapixel color image using matlab on a laptop with $2.6 \mathrm{GHz}$ processor and $4 \mathrm{G}$ RAM.

\section{CONCLUSION}

In this paper, we have proposed an image dehazing method using a pair of RGB and NIR images. The intuition behind this technique is that NIR has deep penetration due to its long wavelength and thus according to the Rayleigh's scattering law, the details of distant objects can be well preserved in the NIR image. We have estimated the airlight color by exploiting the dissimilarity between the RGB and the NIR, and developed an image dehazing procedure to enforce the NIR gradient constraint through an optimization framework. Experimental results on real captured images demonstrate that the proposed image dehazing method unveils the details of the scene with less noise and better color distribution. 


\section{REFERENCES}

[1] S K. Nayar and S G. Narasimhan, "Vision in bad weather," IEEE International Conference on Comput Vision (ICCV), pp. 820-827, 1999.

[2] K M. He, J. Sun, and X O. Tang, "Single image haze removal using dark channel prior," IEEE Conference on Computer Vision and Pattern Recognition (CVPR), 2009.

[3] R. Fattal, "Single image dehazing," ACM Transactions on Graphics (Proc. ACM SIGGRAPH), 2008.

[4] C O. Ancuti, C. Ancuti, C. Hermans, and P. Bekaert, "A fast semi-inverse approach to detect and remove the haze from a single image," 10th Asian Conference on Computer Vision (ACCV), pp. 501-514, 2010.

[5] S. Shwartz, E. Namer, and Y. Schechner, "Blind haze separation," IEEE Conference on Computer Vision and Pattern Recognition (CVPR), 2006.

[6] N. Salamati, A. Germain, and S. Süsstrunk, "Removing shadows from images using color and near-infrared," Proc. IEEE International Conference on Image Processing (ICIP), 2011.

[7] Y M. Lu, C. Fredembach, M. Vetterli, and S. Süsstrunk, "Designing color filter arrays for the joint capture of visible and near-infrared images," Proc. IEEE International Conference on Image Processing (ICIP), 2009.

[8] L. Schaul, C. Fredembach, and S. Süsstrunk, "Color image dehazing using the near-infrared," Proc. IEEE International Conference on Image Processing (ICIP), 2009.

[9] Z. Farbman, R. Fattal, D. Lischinski, and R. Szeliski, "Edge preserving decompositions for multi-scale tone and detail manipulation," International Conference on Computer Graphics and Interactive Techniques, 2008.

[10] K M. He, J. Sun, and X O. Tang, "Guided image filtering," The 11th European Conference on Computer Vision (ECCV), 2010. 